\title{
Complicações de Traumatismo Crânio Encefálico em Paciente com Afibrinogenemia hereditária - Relato de Caso
}

\author{
Complications of Traumatic Brain Injury in Patients with Hereditary Afibrinogenemia - Case \\ Report
}

\author{
Complicaciones de Traumatismo Cráneo Encefálico en Paciente con Afibrinogenemia \\ hereditaria - Reporte de un caso
}

Caroline Cunha da Rocha ${ }^{1 *}$, Caio Nogueira Oliveira ${ }^{1}$, Liter William Pinheiro Nunez ${ }^{1}$, Lucianna Serfaty de Holanda2 $^{2}$, Carla Danielle Nascimento Pontes ${ }^{2}$, Ivy Cavalcante de Almeida e Silva ${ }^{2}$, Ian da Silva Rodrigues ${ }^{2}$, Vitor Bruno Teixeira de Holanda ${ }^{3}$, Alline Neves de Oliveira Mota ${ }^{4}$, Rafaela Mácola Marques ${ }^{5}$, Myriam Ruth da Silva Magalhães ${ }^{5}$, Larissa Nelson Leite ${ }^{6}$, Cristine Bessa Gondim ${ }^{7}$.

\section{RESUMO}

Introdução: A afibrinogenemia é um hereditário do fibrinogênio do tipo I por defeito quantitativo com ausência plasmática total desde. As manifestações clínicas são variadas, podendo apresentar manifestações hemorrágicas leves, moderadas ou graves, assim como trombose. O diagnóstico pode ser suspeitado pelo coagulograma alterado. $O$ tratamento será feito com reposição de fibrinogênio. Detalhamento do caso: Paciente, 6 anos de idade, sexo masculino deu entrada em serviço de Urgência e Emergência pediátrica devida a cefaleia intensa e aumento do perímetro cefálico após trauma em região parietal da cabeça. Menor tem afibrinogenemia hereditária diagnosticada aos 4 meses de vida. Tomografia de crânio mostrou aumento do tecido cutâneo do couro cabeludo de forma simétrica em toda a sua extensão, sem presença de lesão óssea ou acometimento intra-craniano. Paciente realizou tratamento com Crioprecipitado e Fibrinogênio e recebeu alta com quadro melhorado após 6 dias de tratamento e acompanhamento. Discussão: A afibrinogenemia é herdada como caráter autossômico recessivo, o que significa dizer que ambos os pais devem carregar o gene defeituoso para poder transmiti-lo. Após traumas, as crises hemorrágicas podem acontecer precocemente, mas em alguns casos podem ser manterem assintomáticas por longos períodos. Apesar de poder ocorrer hemorragia intracraniana potencialmente fatal, o prognóstico da afibrinogenemia é favorável com o diagnóstico precoce e tratamento adequado. A afibrinogenemia por ser uma doença sem cura e com controle, é de fundamental importância a orientação desde o diagnóstico da doença para os acidentes possam evoluir com trauma e ocasionar sangramentos que levem o paciente a óbito.

Palavras-chave: Afibrinogenemia, Trauma crânio encefálico, Fibrinogênio.

\footnotetext{
ABSTRACT since. The clinical manifestations are varied and may present mild, moderate or severe hemorrhagic

${ }^{1}$ Médica (o) plantonista (o) da Urgência Pediátrica do Pronto Socorro Municipal - Mario Pinotti.

*E-mail: carolinegatti@gmail.com

2 Médica Residente de Clínica Médica da Fundação Santa Casa de Misericórdia do Pará.

${ }^{3}$ Médico Cardiologista do Hospital Saúde da Mulher.

${ }^{4}$ Médica Residente de Dermatologia da Universidade do Estado do Pará.

${ }^{5}$ Médica Residente de Cirurgia Geral do Hospital Universitário João de Barros Barreto.

${ }^{6}$ Médica Pediatra da Urgência Pediátrica do Pronto Socorro Municipal.

${ }^{7}$ Médica Residente de Pediatria da Universidade do Estado do Pará.
}

Introduction: Afibrinogenemia is a hereditary type I fibrinogen by quantitative defect with total plasma absence manifestations, as well as thrombosis. The diagnosis may be suspected by the changed coagulogram. 
Treatment will be made with fibrinogen replacement. Case report: A 6-year-old male patient was admitted to the Emergency and Pediatric Emergency Department due to severe headache and increased head circumference following trauma to the parietal region of the head. Minor has hereditary afibrinogenemia diagnosed at 4 months of age. Skull tomography showed an increase of the cutaneous tissue of the scalp in a symmetrical way in all its extension, without presence of bone lesion or intra-cranial affection. Patient was treated with Cryoprecipitate and Fibrinogen and was discharged with improved condition after 6 days of treatment and follow-up. Discussion: Afibrinogenemia is inherited as an autosomal recessive, which means that both parents must carry the defective gene in order to transmit it. The hemorrhagic episodes, although early, are usually post-traumatic, and the affected individuals may remain asymptomatic for long periods. Although potentially fatal intracranial hemorrhage may occur, the prognosis of afibrinogenemia is favorable with early diagnosis and appropriate treatment. Since afibrinogenemia is a disease without cure and with control, it is of fundamental importance the orientation from the diagnosis of the disease so that the accidents can evolve with trauma and cause bleeding that lead the patient to death.

Key words: Afibrinogenemia, Traumatic brain injury, Fibrinogen.

\section{RESUMEN}

Introducción: El fibrinógeno es un fibrinógeno hereditario de tipo I por defecto cuantitativo con ausencia total de plasma desde entonces. Las manifestaciones clínicas son variadas y pueden presentar manifestaciones hemorrágicas ligero, moderadas o graves, así como trombosis. El diagnóstico puede sospecharse por el cambio de coagulograma. El tratamiento se realizará con reemplazo de fibrinógeno. Reporte de un caso: Un paciente masculino de 6 años ingresó en el Servicio de Urgencias de Emergencia y Pediatría debido a un fuerte dolor de cabeza y una mayor circunferencia de la cabeza luego de un traumatismo en la región parietal de la cabeza. Menor tiene afibrinogenemia hereditaria diagnosticada a los 4 meses de edad. La tomografía de cráneo mostró un aumento del tejido cutáneo del cuero cabelludo de forma simétrica en toda su extensión, sin presencia de lesión ósea ni afecto intracraneal. El paciente fue tratado con Crioprecipitado y Fibrinógeno y fue dado de alta con mejoría luego de 6 días de tratamiento y seguimiento. Discusión: la fibrinogenemia se hereda de forma autosómica recesiva, lo que significa que ambos padres deben portar el gen defectuoso para poder transmitirlo. Los episodios hemorrágicos, aunque temprano, suelen ser postraumáticos y los individuos afectados pueden permanecer asintomáticos durante largos períodos. Aunque puede producirse una hemorragia intracraneal potencialmente mortal, el pronóstico de la afibrinogenemia es favorable con un diagnóstico precoz y un tratamiento apropiado. Dado que la afibrinogenemia es una enfermedad sin cura y con control, es de fundamental importancia la orientación desde el diagnóstico de la enfermedad para que los accidentes puedan evolucionar con trauma y causar sangrado que lleve al paciente a la muerte.

Palabras clave: Afibrinogenemia, Lesión cerebral traumática, Fibrinógeno.

\section{INTRODUÇÃO}

A afibrinogenemia faz parte dos distúrbios hereditários do fibrinogênio do tipo I por defeito quantitativo de fibrinogênio com ausência plasmática total desde (BRASIL, 2015; VU et al, 2003). É uma doença autossômica recessiva rara com prevalência de aproximadamente 1:1.000.000 e atinge ambos os sexos em igual proporção (BRASIL, 2015; PASTILHA et al, 1997). O fibrinogênio é formado por três subunidades (Aa, Bß e Y) codificadas por 3 genes diferentes FGA, FGB e FGG, respectivamente, localizados no braço longo do cromossomo 4 (q28-30), sendo o fibrinogênio o fator de coagulação mais abundante do sangue com concentração plasmática de 160-400mg/dl e meia-vida de 4 dias (BRASIL, 2015; VU et al, 2003; PASTILHA et al, 1997). O fibrinogênio atua no último estágio da coagulação ao ser convertido em fibrina pela trombina, na agregação plaquetária, na fibrinólise e na cicatrização (BRASIL, 2015). 
A afibrinogenemia está associada com sangramento leve a moderado. As manifestações clínicas são variadas, podendo o indivíduo acometido ser assintomático (53\%) ou apresentar manifestações hemorrágicas leves, moderadas ou graves (26\%), assim como trombose (21\%) (BRASIL, 2015; BRASIL, 2016; BOLTONMAGGS, 2006). A associação entre o nível de atividade de coagulação do fibrinogênio pode determinar a gravidade das hemorragias. A afibrinogenemia apresenta hemorragias que acarretam risco à vida e sangramentos mucosos incluindo epistaxes, sangramentos do trato gastrointestinal e do aparelho geniturinário. ${ }^{1,3,5}$ Hemorragias do sistema nervoso central podem ocorrer em até $5 \%$ dos casos, principalmente nas crianças em idade pré-escolar e são as principais causas de óbitos (BRASIL, 2015; FAUCI et all, 2013). Hemartroses e hematomas musculares também são frequentes, mas ocorrem menos do que nas hemofilias A e B grave (BRASIL, 2015).

O diagnóstico pode ser suspeitado pelo prolongamento acentuado do Tempo de protrombina (TP - avalia as vias extrínsecas e comum da coagulação), Tempo de tromboplastina parcial ativada (TTPa - avalia as vias intrínsecas e comum da coagulação) e Tempo de trombina (TT - avalia o tempo em que o fibrinogênio se transforma em fibrina na presença de uma quantidade padrão de trombina), assim como do tempo de reptilase (enzima substrato do veneno da serpente Bothrops que converte fibrinogênio em fibrina por ação direta) que vai estar prolongado, podendo variar também entre a normalidade e encurtado (BRASIL, 2015; (BRASIL, 2016; BOLTON-MAGGS, 2006; CAGNOLATI et al; LORENZI, 2006; GOLDMAN, 2010). Os níveis de fibrinogênio, tanto funcional (Clauss) como antigênico, são indetectáveis (BRASIL, 2015;. (BRASIL, 2016). Apesar da dificuldade em se estabelecer uma correlação genótipo-fenótipo, o diagnóstico definitivo pode ser obtido por meio do estudo de biologia molecular (BRASIL, 2015). Os diagnósticos diferenciais incluem as outras deficiências congênitas de fatores de coagulação (fatores II, V, VII, X, XI, VIII, IX e XIII) e deficiência adquirida de fibrinogênio (coagulopatia de consumo, insuficiência hepática). No caso de trombose, o diagnóstico diferencial inclui também trombofilia congênita ou adquirida (deficiência da antitrombina, deficiência da proteína $\mathrm{C}$ ou $\mathrm{S}$, mutação do fator $\mathrm{V}$ de Leiden, lúpus anticoagulante e mutação $\mathrm{F}$ II de Leiden) (VU et al, 2003; REZENDE, 2010).

O tratamento será feito com reposição de fibrinogênio, sendo para a hemostasia é necessária uma concentração plasmática de $\mathrm{Fl}$ entre $50 \mathrm{mg} / \mathrm{dl}$ e $100 \mathrm{mg} / \mathrm{dl}$ e sua meia-vida é de 2 a 4 dias (BRASIL, 2015; BOLTON-MAGGS, 2006). Antes de repor o fibrinogênio é necessário conhecer a história hemorrágica prévia do paciente. Os produtos que contêm fibrinogênio incluem: plasma fresco congelado (PFC), crioprecipitado e concentrado de fibrinogênio (FI) (BRASIL, 2015; PASTILHA et all, 1997). O FI é oriundo de plasma vírusinativado e é o tratamento padrão-ouro para a reposição nas deficiências do fibrinogênio. Em uma bolsa de sangue contem cerca de $200-250 \mathrm{mg}$ de fibrinogênio e uma unidade $(10 \mathrm{~mL}$ a $15 \mathrm{~mL})$ de crioprecipitado contém o fibrinogênio presente em uma bolsa de sangue. Cada unidade de crioprecipitado aumenta o fibrinogênio plasmático em aproximadamente $7 \mathrm{mg} / \mathrm{dL}$ a $10 \mathrm{mg} / \mathrm{dL}$. Podem ocorrer complicações transfusionais e por isso o crioprecipitado tem sido evitado. Não se recomenda a profilaxia primária nas desordens de fibrinogênio (BRASIL, 2015; PASTILHA et al, 1997). Já a profilaxia secundária é indicada na afibrinogenemia em casos de hemorragias graves e/ou recorrentes, como em sangramento do sistema nervoso central, trato gastrointestinal, hemartroses ou outras que envolvam risco de vida (BRASIL, 2015).

\section{DETALHAMENTO DO CASO}

Paciente, 6 anos de idade, sexo masculino deu entrada em serviço de Urgência e Emergência pediátrica devido a cefaleia intensa e aumento do perímetro cefálico. Menor foi encaminhado pelo Serviço de Referência em Doenças Hematológicas do Estado onde faz acompanhamento devido afibrinogenemia hereditária diagnosticada aos 4 meses de vida. Pai relata que menor há 4 dias da entrada no serviço apresentou queda de mesa com trauma em região parietal e desde então apresentou crescimento do perímetro cefálico, hematoma periorbital direito e cefaleia intensa. Ao exame físico regular estado geral, fascies de dor, eupneico ar ambiente, hipocorado 2+/4+, hidratado, acianótico, anictérico e afebril ao toque. Ausculta cardiológica com 
bulhas normofonéticas, rítmicas e em dois tempos, sem sopro. Ausculta pulmonar com murmúrio vesicular presente bilateralmente, sem ruídos adventícios. Cabeça com edema difuso em couro cabeludo com dor ao toque e aumento do perímetro cefálico e hematoma em periorbital direito. Membros inferior esquerdo com lesão ulcerada e hematomas adjacente (Figura 1A) e membro inferior esquerdo com hematoma em coxa de aproximadamente $12 \mathrm{~cm}$ em seu maior diâmetro (Figura 1B). Iniciado analgesia com acetoaminofeno $15 \mathrm{mg} / \mathrm{kg}$ de seis em seis horas e solicitado avaliação de hematologista do serviço. Tomografia de crânio mostrou aumento do tecido cutâneo do couro cabeludo de forma simétrica em toda a sua extensão, sem presença de lesão óssea ou acometimento intra-craniano (Figura 2A-2B). Hematologista prescreveu fibrinogênio 500mg intra-venoso, $1 \mathrm{vez}$ ao dia, por 2 dias. Enquanto aguardava liberação do Fibrinogênio paciente recebeu Crioprecipitado, 20 unidades ao dia (durante 2 dias). No terceiro dia de internação hospitalar menor evoluiu com hematoma periorbital esquerdo com edema ocular e protrusão orbitária (Figura 3A-3B) sendo avaliado por oftalmologista do serviço descartando comprometimento definitivo de visão. Paciente recebeu fibrinogênio no quarto e quinto dia de internação hospitalar, com melhora definitiva da cefaleia, dos hematomas em membros inferiores e principalmente dos edemas e hematomas periorbitais e do edema em couro cabeludo. Recebeu alta hospitalar com quadro melhorado no sexto dia de internação.

Figura 1: A. Membro inferior esquerdo com lesão ulcerada e hematomas adjacente. B. Membro inferior esquerdo com hematoma em coxa de aproximadamente $12 \mathrm{~cm}$ em seu maior diâmetro.

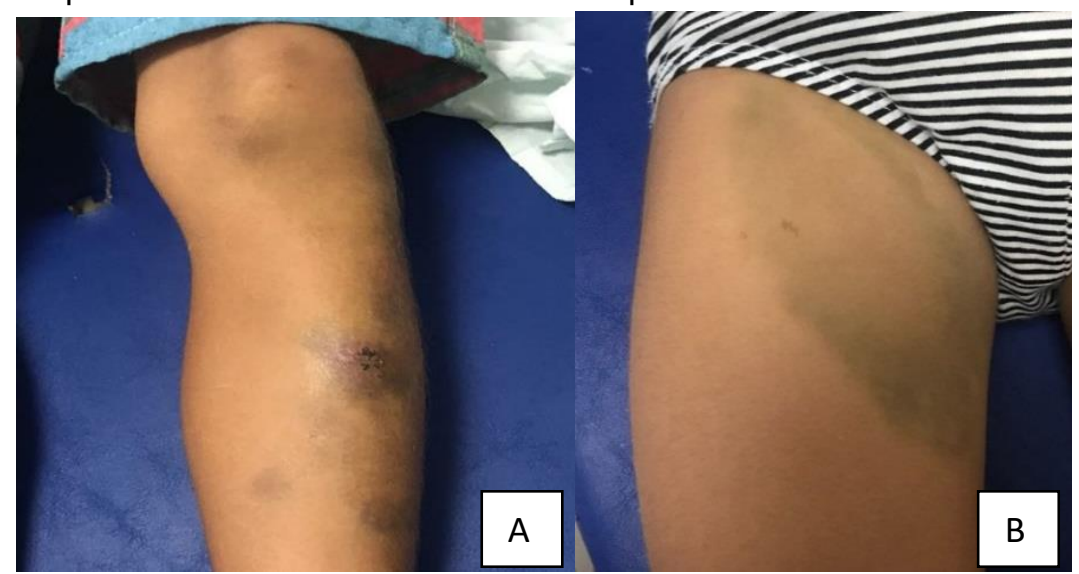

Fonte: Arquivo de prontuário Pronto Socorro Municipal Mario Pinotti.

Figura 2: Tomografia de crânio. A. aumento do tecido cutâneo do couro cabeludo de forma simétrica em toda a sua extensão, sem presença de lesão óssea ou acometimento intra-craniano. B. aumento do tecido cutâneo do couro cabeludo de forma simétrica em toda a sua extensão, sem presença de lesão óssea ou acometimento intra-craniano em corte mais acima que em $\mathrm{A}$.

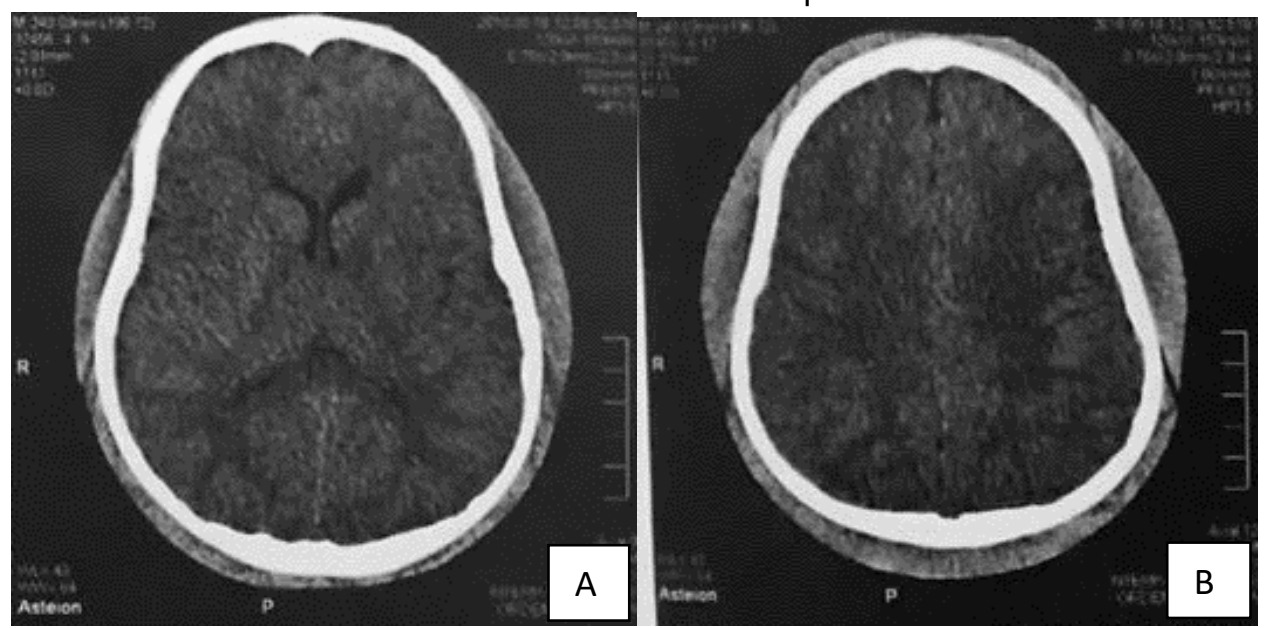

Fonte: Arquivo de prontuário Pronto Socorro Municipal Mario Pinotti. 
Figura 3: terceiro dia de internação hospitalar. A. olho direito evoluindo com hematoma periorbital. B. olho esquerdo com edema ocular e protrusão orbitária.

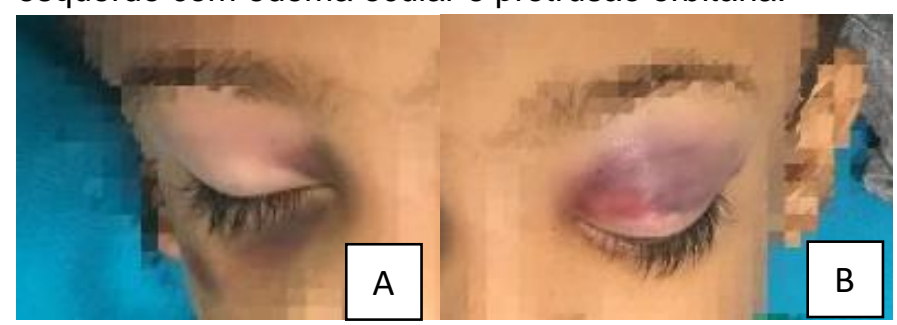

Fonte: Arquivo de prontuário Pronto Socorro Municipal Mario Pinotti.

\section{DISCUSSÃO}

A afibrinogenemia é herdada como caráter autossômico recessivo, o que significa dizer que ambos os pais devem carregar o gene defeituoso para poder transmiti-lo (BRASIL, 2015). Como qualquer outro distúrbio autossômico recessivo, é mais prevalente onde os casamentos consanguíneos são comuns. O paciente já chegou ao nosso serviço com diagnóstico de afibrinogenemia feito pelo centro de referência do Estado não sendo portanto necessário aplicar testes diagnósticos. As hemorragias podem acontecer espontaneamente caracterizadas por sangramento de mucosas, hematomas, hemartroses, sendo geralmente precoce quando associada ao trauma, no paciente em questão, já chegou previamente com sinais de hemorragias previas relatados com surgimento após pequenos associadas a outros traumas que não o que o levou ao atendimento do trauma cranioencefálico, porém em decorrente do trauma evolui com sangramentos que progrediram para hematomas extensos principalmente em região de cabeça (PASTILHA et al, 1997; REZENDE, 2010)

Apesar de poder ocorrer hemorragia intracraniana potencialmente fatal, o prognóstico da afibrinogenemia é favorável com o diagnóstico precoce e tratamento adequado (BRASIL, 2015). O paciente não apresentou nenhuma complicação que resultasse em risco a vida durante o período que permaneceu em internação para a administração do tratamento. Nos casos de hemorragias após eventos traumáticos, o tratamento indicado primeiramente será a estabilização do paciente, analgesia e solicitação de fibrinogênio para repor imediatamente o equilíbrio da hemostasia e na ausência dele o uso de crioprecipitado, assim como foi realizado neste paciente (BRASIL, 2015; (BRASIL, 2016; GOLDMAN, 2010). No primeiro momento o paciente recebeu analgesia, foi prontamente avaliado pelo hematologista do serviço e recebeu em tempo inferior a 24 horas da admissão o crioprecipitado e em menos de 48 horas o fibrinogênio evoluindo com boa resposta terapêutica recebendo alta hospitalar após 6 dias.

Há poucos casos descritos na literatura com associação de Afibronogenemia hereditária e trauma cranioencefálico, embora há citação da importância desta patologia nos eventos com trauma grave e do risco de óbito devido aos eventos hemorrágicos.

\section{REFERÊNCIAS}

1. BRASIL. Ministério da Saúde. Manual das coagulopatias hereditárias raras. Secretaria de Atenção à Saúde, Departamento de Atenção Especializada e Temática. - Brasília: Ministério da Saúde, 2015.

2. VU D, BOLTON-MAGGS PHB, PARR JR, MORRIS MA, et all. Congenital afibrinogenemia: identification and expression of a missense mutation in FGB impairing fibrinogen secretion. Blood, 15 december 2003 volume 102, number 13, 4413-4415.

3. PASTILHA P, COELHO L, COSTA TD, DEUS G, et all. Afibronogenemia congênita. Caso clinico. Acta Médica Portuguesa, 11; 117-1119. 1997.

4. BRASIL. Ministério da Saúde. Manual de diagnóstico laboratorial das Coagulopatias Hereditárias e Plaquetopatias. Secretaria de Atenção à Saúde, Departamento de Atenção Especializada e Temática. - Brasília: Ministério da Saúde, 2016. 
5. BOLTON-MAGGS, PHB. Trastornos de lacoagulación poco comunes. Tratamiento de la hemofilia. Reino Unido. Abril de 2006, No. 39.

6. FAUCI AS, KASPER DL, HAUSER SL, LONGO DL, JAMESON JL. Medicina interna de Harrison. 18.ed. Porto Alegre: AMGH, 2013. 2 v.1. p. 461.

7. CAGNOLATI D, SANKARANKUTTY AK, ROCHA JPS, BEER A, SILVA OC. Hemostasia e distúrbios da coagulação. Faculdade de Medicina de Ribeirão Preto. Acesso: http://sites.usp.br/dcdrp/wpcontent/uploads/sites/273/2017/05/hemostasia revisado.pdf. Acesso em: 26 nov. 2018.

8. LORENZI TF. Manual de Hematologia - Propedêutica e Clínica - 4ª Ed. Guanabara Koogan. 2006, p.501-503

9. GOLDMAN L, CECIL AD. Tratado de Medicina Interna. 23a Ed. Elsevier. 2010. p. 1246-1247.

10. REZENDE SM. Distúrbios da hemostasia: doenças hemorrágicas. Rev Med Minas Gerais 2010; 20(4): 534-553. 